\title{
Extinction and decay estimates of solutions for a $p$-Laplacian evolution equation with nonlinear gradient source and absorption
}

\author{
Xianghui $X u^{1}$ and Zhong Bo Fang ${ }^{2^{*}}$
}

\section{"Correspondence:}

fangzb7777@hotmail.com

${ }^{2}$ School of Mathematical Sciences,

Ocean University of China, Qingdao,

266100, P.R. China

Full list of author information is

available at the end of the article

\begin{abstract}
We investigate the extinction properties of non-negative nontrivial weak solutions of the initial-boundary value problem for a $p$-Laplacian evolution equation with nonlinear gradient source and absorption terms.

MSC: $35 \mathrm{~K} 65 ; 35 \mathrm{~B} 33 ; 35 \mathrm{~B} 40$
\end{abstract}

Keywords: $p$-Laplacian evolution equation; extinction; nonextinction; decay estimate

\section{Introduction}

We are concerned with the initial-boundary value problem for a $p$-Laplacian evolution equation with gradient source and absorption terms,

$$
\begin{aligned}
& u_{t}=\operatorname{div}\left(|\nabla u|^{p-2} \nabla u\right)+\lambda|\nabla u|^{r}-\beta u^{q}, \quad x \in \Omega, t>0, \\
& u(x, t)=0, \quad x \in \partial \Omega, t>0, \\
& u(x, 0)=u_{0}(x), \quad x \in \Omega,
\end{aligned}
$$

where $1<p<2,0<q \leq 1, \lambda, \beta, r>0, \Omega \subset R^{N}(N \geq 1)$ is a bounded domain with smooth boundary and $u_{0}(x) \in L^{\infty}(\Omega) \cap W_{0}^{1, p}(\Omega)$ is a non-negative function. The symbols $\|\cdot\|_{s}$, $\|\cdot\|_{1, s}$ denote $L^{s}(\Omega), W^{1, s}(\Omega)$ norms, respectively (where $s \geq 1$ ), and $|\Omega|$ denotes the measure of $\Omega$.

Equation (1.1) appears in the study of non-Newtonian fluids through porous media, combustion theory, and the turbulent flow of a gas in porous medium. In the nonNewtonian fluid theory, the quantity $p$ is a characteristic of the medium. Media with $p>2$ are called dilatant fluids and those with $p<2$ are called pseudo plastics. If $p=2$, they are Newtonian fluids. The $p$-Laplacian operator also appears in the study of torsional creep (elastic for $p=2$, plastic as $p<2$, see [1]), flow through porous media $\left(p=\frac{3}{2}\right.$, see [2]) or glacial sliding $\left(p \in\left(1, \frac{4}{3}\right]\right.$, see [3]). Many nonlinear diffusion phenomena are described by the cooperation and interaction between the nonlinear source term and absorption term during the diffusion. From a physical point of view, $\lambda|\nabla u|^{r}$ is called gradient source term and $-\beta u^{q}$ represents an absorption term.

The extinction phenomenon is an important property for solutions of many evolutionary equations, especially for fast diffusion equations. In 1974, Kalashnikov [4] consid- 
ered the Cauchy problem of a semilinear equation with absorption term $u_{t}=\Delta u-u^{q}$ and firstly introduced the definition of extinction for its solution, that is, there exists a finite time $T>0$ such that the solution is nontrivial on $(0, T)$ and then $u(x, t) \equiv 0$ for all $(x, t) \in \Omega \times[T,+\infty)$. In this case, $T$ is called the extinction time. Later, many authors became interested in the extinction and nonextinction of all kinds of evolutionary equations. We have the following parabolic equation without gradient source term:

$$
u_{t}=\operatorname{div}\left(|\nabla u|^{p-2} \nabla u\right)+\lambda u^{r}-\beta u^{q}, \quad x \in \Omega, t>0,
$$

where $r>0$ and $0<q \leq 1$. In the case $\lambda=\beta=0$, Dibenedetto [5] and Yuan et al. [6] proved that the necessary and sufficient condition for the extinction to occur is $1<p<2$. For the case $\lambda=0, \mathrm{Gu}$ [7] proved that if $1<p<2$ or $0<q<1$, the solutions of the problem vanish in finite time, but if $p \geq 2$ and $q \geq 1$, there is nonextinction. Tian [8] and Yin et al. [9] showed that $r=p-1$ is the critical exponent of the weak solution for the case $\beta=0$. But all the results are limited to a local range and a higher dimensional space, while a precise decay estimate has not been given. Recently, Fang and Li [10] considered equation (1.4) with $q=1$, when the diffusion term was replaced by a doubly degenerate operator in the whole dimensional space, and they showed that the extinction of the weak solution is determined by competition of source and absorption terms. They also obtained the exponential decay estimates which depend on the initial data, coefficients, and domains. Thereafter, they obtained the same results for a class of nonlocal problems, see [11, 12].

Recently, many researchers have devoted studies to the occurrence of such a phenomenon for a class of nonlinear parabolic equations with gradient terms. For example, Benachour et al. [13] considered the semilinear heat equation with absorption term,

$$
u_{t}=\Delta u-\lambda|\nabla u|^{r}, \quad x \in \Omega, t>0
$$

subject to (1.2) and (1.3) and proved that the sufficient condition for the extinction to occur is $0<r<1$ by using the upper and lower solutions methods. Lagar et al. [14] studied the qualitative properties of non-negative solutions to the Cauchy problem for the fast diffusion equation with gradient absorption

$$
u_{t}=\operatorname{div}\left(|\nabla u|^{p-2} \nabla u\right)-|\nabla u|^{q}, \quad x \in R^{N}, t>0,
$$

with $1<p<2, q>0$ and obtained the result that the solution of (1.6) either remains positive as $t \rightarrow \infty$ for $q>p-\frac{N}{N+1}$ or vanishes in finite time for $0<q<\frac{p}{2}$. For the porous medium equation with gradient source term and without absorption term, the research of the extinction and nonextinction of solutions has also been performed (see [15]).

Motivated by the works mentioned above, and because there is little literature on the study of the extinction and nonextinction properties for parabolic equations with nonlinear gradient source and absorption terms, in this paper, our goal is to establish the sufficient conditions about the extinction and nonextinction of solutions for the problem (1.1)(1.3) in the whole dimensional space. By combining the $L^{p}$-integral norm estimate method and the technique of differential inequalities, we find that the critical exponent of extinction for the non-negative weak solution is determined by the competition of nonlinear terms for $1<p<2$, and decay estimates depend on the choices of initial data, coefficients, and domain. More precisely, we obtain the following results. 
Theorem 1 Assume that $1<p<2, q=1$ and $p-1=r$.

(1) If $N \geq 2$, the non-negative weak solution of problem (1.1)-(1.3) vanishes in finite time for any non-negative initial data $u_{0}$ provided that $\lambda$ is sufficiently small, and we have the following.

(a) If $\frac{2 N}{N+2}<p<2$,

$$
\begin{aligned}
& \|u(\cdot, t)\|_{2} \leq\left[\left(\left\|u_{0}\right\|_{2}^{2-p}+\frac{C_{1}}{\beta}\right) e^{(p-2) \beta t}-\frac{C_{1}}{\beta}\right]^{\frac{1}{2-p}}, \quad t \in\left[0, T_{1}\right), \\
& \|u(\cdot, t)\|_{2} \equiv 0, \quad t \in\left[T_{1},+\infty\right) .
\end{aligned}
$$

(b) If $1<p \leq \frac{2 N}{N+2}$,

$$
\begin{aligned}
& \|u(\cdot, t)\|_{l+1} \leq\left[\left(\left\|u_{0}\right\|_{l+1}^{2-p}+\frac{C_{2}}{\beta}\right) e^{(p-2) \beta t}-\frac{C_{2}}{\beta}\right]^{\frac{1}{2-p}}, \quad t \in\left[0, T_{2}\right), \\
& \|u(\cdot, t)\|_{l+1} \equiv 0, \quad t \in\left[T_{2},+\infty\right) .
\end{aligned}
$$

Here $l=\frac{2 N-(N+1) p}{p}, C_{1}, T_{1}, C_{2}$, and $T_{2}$ are given by (3.5), (3.6), (3.11), and (3.12), respectively.

(2) If $N=1$, the non-negative weak solution of problem (1.1)-(1.3) vanishes in finite time for any non-negative initial data $u_{0}$ provided that $\lambda$ is sufficiently small, and

$$
\begin{aligned}
& \|u(\cdot, t)\|_{2} \leq\left[\left(\left\|u_{0}\right\|_{2}^{2-p}+\frac{C_{3}}{\beta}\right) e^{(p-2) \beta t}-\frac{C_{3}}{\beta}\right]^{\frac{1}{2-p}}, \quad t \in\left[0, T_{3}\right), \\
& \|u(\cdot, t)\|_{2} \equiv 0, \quad t \in\left[T_{3},+\infty\right),
\end{aligned}
$$

where $C_{3}$ and $T_{3}$ are given by (3.15) and (3.16), respectively.

Theorem 2 Assume that $1<p<2, q=1$ and $p-1<r<\frac{p}{2}$.

(1) If $N \geq 2$, the non-negative weak solution of problem (1.1)-(1.3) vanishes in finite time provided that $u_{0}$ (or $\lambda$ or $|\Omega|$ ) is sufficiently small, and we have the following.

(a) If $\frac{2 N}{N+2}<p<2$,

$$
\begin{aligned}
& \|u(\cdot, t)\|_{2} \leq\left\|u_{0}\right\|_{2} e^{-\alpha_{1} t}, \quad t \in\left[0, T_{4}\right), \\
& \|u(\cdot, t)\|_{2} \leq\left[\left(\left\|u\left(\cdot, T_{4}\right)\right\|_{2}^{2-p}+\frac{C_{4}}{\beta}\right) e^{(p-2) \beta\left(t-T_{4}\right)}-\frac{C_{4}}{\beta}\right]^{\frac{1}{2-p}}, \quad t \in\left[T_{4}, T_{5}\right), \\
& \|u(\cdot, t)\|_{2} \equiv 0, \quad t \in\left[T_{5},+\infty\right) .
\end{aligned}
$$

(b) If $1<p \leq \frac{2 N}{N+2}$,

$$
\begin{aligned}
\|u(\cdot, t)\|_{l+1} & \leq\left\|u_{0}\right\|_{l+1} e^{-\alpha_{2} t}, \quad t \in\left[0, T_{6}\right), \\
\|u(\cdot, t)\|_{l+1} & \leq\left[\left(\left\|u\left(\cdot, T_{6}\right)\right\|_{l+1}^{2-p}+\frac{C_{5}}{\beta}\right) e^{(p-2) \beta\left(t-T_{6}\right)}-\frac{C_{5}}{\beta}\right]^{\frac{1}{2-p}}, \quad t \in\left[T_{6}, T_{7}\right), \\
\|u(\cdot, t)\|_{l+1} & \equiv 0, \quad t \in\left[T_{7},+\infty\right),
\end{aligned}
$$

where $l=\frac{2 N-(N+1) p}{p}, C_{4}, T_{5}, C_{5}$, and $T_{7}$ are given by (3.17)-(3.20), respectively. 
(2) If $N=1$, the non-negative weak solution of problem (1.1)-(1.3) vanishes in finite time provided that $u_{0}$ (or $\lambda$ or $\left.|\Omega|\right)$ is sufficiently small, and

$$
\begin{aligned}
\|u(\cdot, t)\|_{2} & \leq\left\|u_{0}\right\|_{2} e^{-\alpha_{3} t}, \quad t \in\left[0, T_{8}\right), \\
\|u(\cdot, t)\|_{2} & \leq\left[\left(\left\|u\left(\cdot, T_{8}\right)\right\|_{2}^{2-p}+\frac{C_{6}}{\beta}\right) e^{(p-2) \beta\left(t-T_{8}\right)}-\frac{C_{6}}{\beta}\right]^{\frac{1}{2-p}}, \quad t \in\left[T_{8}, T_{9}\right), \\
\|u(\cdot, t)\|_{2} & \equiv 0, \quad t \in\left[T_{9},+\infty\right),
\end{aligned}
$$

where $C_{6}$ and $T_{9}$ are given by (3.21) and (3.22), respectively.

Theorem 3 Assume $1<p<2, q=1$ and $p-1>r$. Then the non-negative weak solution of problem (1.1)-(1.3) cannot vanish in finite time for any non-negative initial data $u_{0}$ provided that $\lambda$ is sufficiently large.

Remark 1 According to Theorems 1-3, we observe that $p-1=r$ is still the critical exponent of extinction for the solution of (1.1)-(1.3) when $1<p<2$ and $q=1$.

Remark 2 Assume that $p \geq 2, q=1$, then the non-negative weak solution of problem (1.1)(1.3) cannot vanish in finite time for any $r>0$ and non-negative initial data. This result can be extended to the case $q \geq 1$ (the detailed proof can be found in [7]).

Theorem 4 Assume that $1<p<2,0<q<1$ and $p-1=r$, the non-negative weak solution of problem (1.1)-(1.3) vanishes in finite time for any non-negative initial data $u_{0}$ provided that $\lambda$ is sufficiently small.

Theorem 5 Assume that $1<p<2,0<q<1$.

(1) If $N \geq 2, \frac{2 N}{N+2}<p<2$ with $\frac{2 p^{2} q+N p(p-q-1)}{2 p(1+q)+2 N(p-q-1)}<r<\frac{p}{2}$ or $1<p \leq \frac{2 N}{N+2}$ with $\frac{p^{2} q(s+1)+N p(p-q-1)}{p(s+1)(1+q)+2 N(p-q-1)}<r<\frac{p}{2}$, the non-negative weak solution of problem (1.1)-(1.3) vanishes in finite time provided that $u_{0}$ (or $\lambda$ or $|\Omega|$ ) is sufficiently small or $\beta$ is sufficiently large (where $\left.s>\frac{2 N-(N+1) p}{p}\right)$.

(2) If $N=1, \frac{2 p^{2} q+N p(p-q-1)}{2 p(1+q)+2 N(p-q-1)}<r<\frac{p}{2}$, the non-negative weak solution of problem (1.1)-(1.3) vanishes in finite time provided that $u_{0}($ or $\lambda$ or $|\Omega|)$ is sufficiently small or $\beta$ is sufficiently large.

Remark 3 If $p-1 \leq q<1$, the conditions in Theorem 5 imply that $r>p-1$.

Remark 4 Assume that $p=2,0<q<1$; Theorem 4 and Theorem 5 are still established.

Remark 5 Assume that $p>2,0<q<1$ and $\frac{2 p^{2} q+N p(p-q-1)}{2 p(1+q)+2 N(p-q-1)}<r<\frac{p}{2}$; the non-negative weak solution of problem (1.1)-(1.3) vanishes in finite time provided that $u_{0}$ (or $\lambda$ or $|\Omega|$ ) is sufficiently small or $\beta$ is sufficiently large (as $\frac{2 N}{N+2}<2<p$, the proof of this result is the same as the proof for the case $\frac{2 N}{N+2}<p<2$ in Theorem 5(1)).

Remark 6 Assume that $p>2,0<q<1, \frac{p q}{1+q}<r \leq \frac{p^{2} q+N(p-1)(p-q-1)}{p(q+1)+N(p-q-1)}$ and $r<\frac{p}{2}$, the nonnegative weak solution of problem (1.1)-(1.3) vanishes in finite time for any non-negative initial data $u_{0}$ provided that $\lambda$ (or $|\Omega|$ ) is sufficiently small or $\beta$ is sufficiently large (the detailed proof can be referred to [16]). 
Remark 7 If $r=\frac{p}{2}$, Theorem 2, Theorem 5, and Remark 5 will be still established and the choice of $|\Omega|$ will not affect the extinction behavior of solutions any longer.

Remark 8 Theorems 1-5 all require that $u_{0}$ or $\lambda$ or $|\Omega|$ should be sufficiently small or $\beta$ should be sufficiently large, and we will give more concrete conditions to satisfy in the later proofs.

The outline of the paper is as follows. In Section 2, we firstly give the definition of weak solutions for problem (1.1)-(1.3), and then give some preliminary lemmas. Then we prove Theorems 1-3 and Theorems 4-5 in Section 3 and Section 4, respectively.

\section{Preliminary results}

Due to the singularity of (1.1), problem (1.1)-(1.3) has no classical solutions in general, and hence it is reasonable to find a weak solution of the problem. To this end, we first give the following definition of a weak local solution.

Definition 1 We say that a non-negative nontrivial function $u(x, t)$ defined in $Q_{T}=\Omega \times$ $(0, T)$ is a weak solution of problem (1.1)-(1.3) if the following conditions hold:

(i) $u \in C\left(0, T ; L^{\infty}(\Omega)\right) \cap L^{p}\left(0, T ; W_{0}^{1, p}(\Omega)\right), u_{t} \in L^{2}\left(0, T ; L^{2}(\Omega)\right)$;

(ii) For any $0<t_{1}<t_{2}<T$ and any test function $0 \leq \varphi \in C_{0}^{\infty}\left(Q_{T}\right)$

$$
\begin{aligned}
\int_{\Omega} u\left(x, t_{2}\right) \varphi\left(x, t_{2}\right) d x= & \int_{\Omega} u\left(x, t_{1}\right) \varphi\left(x, t_{1}\right) d x+\int_{t_{1}}^{t_{2}} \int_{\Omega}\left\{u \varphi_{s}-|\nabla u|^{p-2} \nabla u \cdot \nabla \varphi\right\} d x d s \\
& +\int_{t_{1}}^{t_{2}} \int_{\Omega}\left\{\lambda|\nabla u|^{r}-\beta u^{q}\right\} \varphi(x, s) d x d s
\end{aligned}
$$

(iii) $u(x, t)=u_{0}(x)$ a.e. $x \in \Omega$.

We can also define the weak lower solution and the weak upper solution of problem (1.1)-(1.3) in the same way except that the ' $=$ ' in Definition 1 is replaced by ' $\leq$ ' and ' $\geq$ ', respectively. Similar to the analysis in [17] and [14, Section 6], the existence in time of a non-negative weak local solution of problem (1.1)-(1.3) can be constructed by the usual vanishing viscosity method which would satisfy a comparison principle.

Before proving our main results, we show some preliminary lemmas and the GagliardoNirenberg inequality which are very important in the following proofs of our results. As for the proofs of these lemmas, we will not repeat them again (see $[10-12,18])$.

Lemma 1 Let $y(t)$ be a non-negative absolutely continuous function on $[0,+\infty)$ satisfying

$$
\frac{d y}{d t}+\alpha y^{k} \leq 0, \quad t \geq 0 ; \quad y(0) \geq 0
$$

where $\alpha>0$ is a constant and $k \in(0,1)$, then we have the decay estimate

$$
\begin{aligned}
& y(t) \leq\left[y^{1-k}(0)-\alpha(1-k) t\right]^{\frac{1}{1-k}}, \quad t \in\left[0, T_{*}\right), \\
& y(t) \equiv 0, \quad t \in\left[T_{*},+\infty\right)
\end{aligned}
$$

where $T_{*}=\frac{y^{1-k}(0)}{\alpha(1-k)}$. 
Lemma 2 Let $y(t)$ be a non-negative absolutely continuous function on $[0,+\infty)$ satisfying

$$
\frac{d y}{d t}+\alpha y^{k}+\beta y \leq 0, \quad t \geq T_{0} ; \quad y\left(T_{0}\right) \geq 0,
$$

where $\alpha, \beta>0$ are constants and $k \in(0,1)$, then we have the decay estimate

$$
\begin{aligned}
& y(t) \leq\left[\left(y^{1-k}\left(T_{0}\right)+\frac{\alpha}{\beta}\right) e^{(k-1) \beta\left(t-T_{0}\right)}-\frac{\alpha}{\beta}\right]^{\frac{1}{1-k}}, \quad t \in\left[T_{0}, T_{*}\right), \\
& y(t) \equiv 0, \quad t \in\left[T_{*},+\infty\right),
\end{aligned}
$$

where $T_{*}=\frac{1}{(1-k) \beta} \ln \left(1+\frac{\beta}{\alpha} y^{1-k}\left(T_{0}\right)\right)+T_{0}$.

Lemma 3 Let $0<k<m \leq 1, y(t) \geq 0$ be a solution of the differential inequality

$$
\frac{d y}{d t}+\alpha y^{k}+\beta y \leq \gamma y^{m}, \quad t \geq 0 ; \quad y(0)=y_{0}>0,
$$

where $\alpha, \beta>0, \gamma$ is a positive constant such that $\gamma<\alpha y_{0}^{k-m}$, then there exists $\eta>\beta$, such that

$$
0 \leq y(t) \leq y_{0} e^{-\eta t}, \quad t \geq 0 .
$$

Lemma 4 Let $\alpha, \beta, \gamma>0$ and $0<m<k<1$, then there exists at least one non-constant solution of the ODE problem

$$
\frac{d y}{d t}+\alpha y^{k}+\beta y=\gamma y^{m}, \quad t \geq 0 ; \quad y(0)=y_{0}>0, \quad y(t)>0, t>0 .
$$

Lemma 5 (Gagliardo-Nirenberg inequality) Suppose that $u \in W_{0}^{k, m}(\Omega), 1 \leq m \leq+\infty, 0 \leq$ $j<k, 1 \geq \frac{1}{r} \geq \frac{1}{m}-\frac{k}{N}$, then we have

$$
\left\|D^{j} u\right\|_{q} \leq C\left\|D^{k} u\right\|_{m}^{\theta}\|u\|_{r}^{1-\theta}
$$

where $C$ is a constant depending only on $N, m, r, j, k, q$ and $\frac{1}{q}=\frac{j}{N}+\theta\left(\frac{1}{m}-\frac{k}{N}\right)+\frac{1-\theta}{r}, 0 \leq \theta<1$. If $m<\frac{N}{k-j}$, then $q \in\left[\frac{N r}{N+r j}, \frac{N m}{N-(k-j) m}\right]$, if $m \geq \frac{N}{k-j}$, then $q \in\left[\frac{N r}{N+r j},+\infty\right]$.

\section{The case $1<p<2, q=1$}

\subsection{Proof of Theorem 1}

(1) If $N \geq 2$, we have the following.

(a) If $\frac{2 N}{N+2}<p<2$, multiplying (1.1) by $u$ and integrating over $\Omega$ yield

$$
\frac{1}{2} \frac{d}{d t}\|u\|_{2}^{2}+\|\nabla u\|_{p}^{p}+\beta\|u\|_{2}^{2}=\lambda \int_{\Omega} u|\nabla u|^{r} d x
$$


since $p-1=r$, we can easily get $\frac{r}{p-r}=p-1<1$. By the Young inequality, we obtain

$$
\begin{aligned}
\int_{\Omega} u|\nabla u|^{r} d x & \leq \varepsilon\|\nabla u\|_{p}^{p}+C(\varepsilon)\|u\|_{1+\frac{r}{p-r}}^{1+\frac{r}{p-r}} \\
& \leq \varepsilon\|\nabla u\|_{p}^{p}+C(\varepsilon)|\Omega|^{\frac{p-2 r}{2(p-r)}}\|u\|_{2}^{1+\frac{r}{p-r}} \\
& =\varepsilon\|\nabla u\|_{p}^{p}+C(\varepsilon)|\Omega|^{\frac{2-p}{2}}\|u\|_{2}^{p}
\end{aligned}
$$

substituting (3.2) into (3.1) leads to

$$
\frac{1}{2} \frac{d}{d t}\|u\|_{2}^{2}+(1-\lambda \varepsilon)\|\nabla u\|_{p}^{p}+\beta\|u\|_{2}^{2} \leq \lambda C(\varepsilon)|\Omega|^{\frac{2-p}{2}}\|u\|_{2}^{p}
$$

Here we can choose $\varepsilon$ small enough such that $1-\lambda \varepsilon>0$. By the Hölder inequality and the Sobolev embedding inequality, we have

$$
\|u\|_{2} \leq|\Omega|^{\frac{1}{2}-\frac{N-p}{N p}}\|u\|_{\frac{N p}{N-p}} \leq C_{0}|\Omega|^{\frac{1}{2}-\frac{N-p}{N p}}\|\nabla u\|_{p}
$$

then we substitute (3.3) into (3.1) to obtain

$$
\frac{1}{2} \frac{d}{d t}\|u\|_{2}^{2}+\left[(1-\lambda \varepsilon) C_{0}^{-p}|\Omega|^{\frac{N-p}{N}-\frac{p}{2}}-\lambda C(\varepsilon)|\Omega|^{\frac{2-p}{2}}\right]\|u\|_{2}^{p}+\beta\|u\|_{2}^{2} \leq 0
$$

i.e.

$$
\frac{d}{d t}\|u\|_{2}+C_{1}\|u\|_{2}^{p-1}+\beta\|u\|_{2} \leq 0
$$

where

$$
C_{1}=(1-\lambda \varepsilon) C_{0}^{-p}|\Omega|^{\frac{N-p}{N}-\frac{p}{2}}-\lambda C(\varepsilon)|\Omega|^{\frac{2-p}{2}}
$$

Once $\varepsilon$ is fixed, we can choose $\lambda$ small enough such that $C_{1}>0$. By Lemma 2 , we can obtain the desired decay estimate for

$$
T_{1}=\frac{1}{(2-p) \beta} \ln \left(1+\frac{\beta}{C_{1}}\left\|u_{0}\right\|_{2}^{2-p}\right)
$$

(b) If $1<p \leq \frac{2 N}{N+2}$, multiplying (1.1) by $u^{l}\left(l=\frac{2 N-(N+1) p}{p} \geq 1\right)$ and integrating over $\Omega$ yield

$$
\frac{1}{l+1} \frac{d}{d t}\|u\|_{l+1}^{l+1}+\frac{l p^{p}}{(p+l-1)^{p}}\left\|\nabla u^{\frac{p+l-1}{p}}\right\|_{p}^{p}+\beta\|u\|_{l+1}^{l+1}=\lambda \int_{\Omega} u^{l}|\nabla u|^{r} d x .
$$

By the Young inequality, we have

$$
\begin{aligned}
\int_{\Omega} u^{l}|\nabla u|^{r} d x & =\left(\frac{p+l-1}{p}\right)^{r} \int_{\Omega} u^{l-\frac{(l-1) r}{p}}\left|\nabla u^{\frac{p+l-1}{p}}\right|^{r} d x \\
& \leq \varepsilon\left\|\nabla u^{\frac{p+l-1}{p}}\right\|_{p}^{p}+C(\varepsilon) \int_{\Omega} u^{l+\frac{r}{p-r}} d x
\end{aligned}
$$




$$
\begin{aligned}
& \leq \varepsilon\left\|\nabla u^{\frac{p+l-1}{p}}\right\|_{p}^{p}+C(\varepsilon)|\Omega|^{\frac{p-2 r}{(l+1)(p-r)}}\|u\|_{l+1}^{l+\frac{r}{p-r}} \\
& =\varepsilon\left\|\nabla u^{\frac{p+l-1}{p}}\right\|_{p}^{p}+C(\varepsilon)|\Omega|^{\frac{1-r}{l+1}}\|u\|_{l+1}^{l+p-1},
\end{aligned}
$$

then we substitute (3.8) into (3.7) to get

$$
\begin{aligned}
& \frac{1}{l+1} \frac{d}{d t}\|u\|_{l+1}^{l+1}+\left[\frac{l p^{p}}{(p+l-1)^{p}}-\lambda \varepsilon\right]\left\|\nabla u^{\frac{p+l-1}{p}}\right\|_{p}^{p}+\beta\|u\|_{l+1}^{l+1} \\
& \quad \leq \lambda C(\varepsilon)|\Omega|^{\frac{1-r}{l+1}}\|u\|_{l+1}^{l+p-1} .
\end{aligned}
$$

Here we can choose $\varepsilon$ small enough such that $\frac{l p^{p}}{(p+l-1)^{p}}-\lambda \varepsilon>0$. By the Sobolev embedding inequality, we have

$$
\left\|u^{\frac{p+l-1}{p}}\right\|_{\frac{N p}{N-p}} \leq C_{00}\left\|\nabla u^{\frac{p+l-1}{p}}\right\|_{p}
$$

i.e.

$$
C_{00}^{-p}\|u\|_{\frac{(p+l-1) N}{N-p}}^{p+l-1} \leq\left\|\nabla u^{\frac{p+l-1}{p}}\right\|_{p}^{p}
$$

By the choice of $l$, we have

$$
C_{00}^{-p}\|u\|_{l+1}^{p+l-1} \leq\left\|\nabla u^{\frac{p+l-1}{p}}\right\|_{p}^{p} .
$$

Substituting (3.10) into (3.9) leads to

$$
\frac{d}{d t}\|u\|_{l+1}+C_{2}\|u\|_{l+1}^{p-1}+\beta\|u\|_{l+1} \leq 0
$$

where

$$
C_{2}=C_{00}^{-p}\left[\frac{l p^{p}}{(p+l-1)^{p}}-\lambda \varepsilon\right]-\lambda C(\varepsilon)|\Omega|^{\frac{1-r}{l+1}} .
$$

Once $\varepsilon$ is fixed, we can choose $\lambda$ small enough such that $C_{2}>0$. By Lemma 2, we can obtain the desired decay estimate for

$$
T_{2}=\frac{1}{(2-p) \beta} \ln \left(1+\frac{\beta}{C_{2}}\left\|u_{0}\right\|_{l+1}^{2-p}\right) .
$$

(2) If $N=1$, multiplying (1.1) by $u^{k}$ and integrating over $\Omega$, and then using the Young inequality, we have

$$
\begin{aligned}
& \frac{1}{k+1} \frac{d}{d t}\|u\|_{k+1}^{k+1}+\left[\frac{k p^{p}}{(k+p-1)^{p}}-\lambda \varepsilon\right]\left\|\nabla u^{\frac{p+k-1}{p}}\right\|_{p}^{p}+\beta\|u\|_{k+1}^{k+1} \\
& \quad \leq \lambda C(\varepsilon)|\Omega| \frac{1-r}{k+1}\|u\|_{k+1}^{k+p-1} .
\end{aligned}
$$

By the Sobolev embedding theorem, we have

$$
\left\|u^{\frac{p+k-1}{p}}\right\|_{d} \leq \gamma\left\|\nabla u^{\frac{p+k-1}{p}}\right\|_{p}
$$


i.e.

$$
\gamma^{-p}\|u\|_{\frac{(p+k-1) d}{p}}^{p+k-1} \leq\left\|\nabla u^{\frac{p+k-1}{p}}\right\|_{p}^{p}
$$

where $\gamma>0, d \geq p$. Here setting $k=1, d=2$ leads to

$$
\frac{d}{d t}\|u\|_{2}+C_{3}\|u\|_{2}^{p-1}+\beta\|u\|_{2} \leq 0,
$$

where

$$
C_{3}=(1-\lambda \varepsilon) \gamma^{-p}-\lambda C(\varepsilon)|\Omega|^{\frac{1-r}{2}} .
$$

Once $\varepsilon$ is fixed, we can choose $\lambda$ small enough such that $C_{3}>0$. By Lemma 2, we can obtain the desired decay estimate for

$$
T_{3}=\frac{1}{(2-p) \beta} \ln \left(1+\frac{\beta}{C_{3}}\left\|u_{0}\right\|_{2}^{2-p}\right) .
$$

\subsection{Proof of Theorem 2}

(1) If $N \geq 2$, we have the following.

(a) If $\frac{2 N}{N+2}<p<2$, multiplying (1.1) by $u$ and integrating over $\Omega$ yield

$$
\frac{1}{2} \frac{d}{d t}\|u\|_{2}^{2}+\|\nabla u\|_{p}^{p}+\beta\|u\|_{2}^{2}=\lambda \int_{\Omega} u|\nabla u|^{r} d x,
$$

and substituting (3.2) and (3.3) into the above equality gives

$$
\frac{1}{2} \frac{d}{d t}\|u\|_{2}^{2}+(1-\lambda \varepsilon) C_{0}^{-p}|\Omega|^{\frac{N-p}{N}-\frac{p}{2}}\|u\|_{2}^{p}+\beta\|u\|_{2}^{2} \leq \lambda C(\varepsilon)|\Omega|^{\frac{p-2 r}{(2 p-r)}}\|u\|_{2}^{1+\frac{r}{p-r}},
$$

i.e.

$$
\frac{d}{d t}\|u\|_{2}+(1-\lambda \varepsilon) C_{0}^{-p}|\Omega|^{\frac{N-p}{N}-\frac{p}{2}}\|u\|_{2}^{p-1}+\beta\|u\|_{2} \leq \lambda C(\varepsilon)|\Omega|^{\frac{p-2 r}{2(p-r)}}\|u\|_{2}^{\frac{r}{p-r}} .
$$

By Lemma 3, there exists $\alpha_{1}>\beta$ such that

$$
0 \leq\|u\|_{2} \leq\left\|u_{0}\right\|_{2} e^{-\alpha_{1} t}, \quad t \geq 0,
$$

provided that

$$
\left\|u_{0}\right\|_{2}<\left[\frac{(1-\lambda \varepsilon) C_{0}^{-p}|\Omega|^{\frac{N-p}{N}-\frac{p}{2}}}{\lambda C(\varepsilon)|\Omega|^{\frac{p-2 r}{2(p-r)}}}\right]^{\frac{1}{p-r}-p+1} .
$$

Furthermore, there exists $T_{4}>0$ such that

$$
\begin{aligned}
& (1-\lambda \varepsilon) C_{0}^{-p}|\Omega|^{\frac{N-p}{N}-\frac{p}{2}}-\lambda C(\varepsilon)|\Omega|^{\frac{p-2 r}{2(p-r)}}\|u\|_{2}^{\frac{r}{p-r}-p+1} \\
& \quad \geq(1-\lambda \varepsilon) C_{0}^{-p}|\Omega|^{\frac{N-p}{N}-\frac{p}{2}}-\lambda C(\varepsilon)|\Omega|^{\frac{p-2 r}{2(p-r)}}\left(\left\|u_{0}\right\|_{2} e^{-\alpha_{1} T_{4}}\right)^{\frac{r}{p-r}-p+1}=C_{4}>0
\end{aligned}
$$


holds for $t \in\left[T_{4},+\infty\right)$. Therefore, when $t \in\left[T_{4},+\infty\right)$, we have

$$
\frac{d}{d t}\|u\|_{2}+C_{4}\|u\|_{2}^{p-1}+\beta\|u\|_{2} \leq 0
$$

By Lemma 2, we can obtain the desired decay estimate for

$$
T_{5}=\frac{1}{(2-p) \beta} \ln \left(1+\frac{\beta}{C_{4}}\left\|u\left(\cdot, T_{4}\right)\right\|_{2}^{2-p}\right)+T_{4} .
$$

(b) If $1<p \leq \frac{2 N}{N+2}$, multiplying (1.1) by $u^{l}\left(l=\frac{2 N-(N+1) p}{p} \geq 1\right)$ and integrating over $\Omega$ yield

$$
\frac{1}{l+1} \frac{d}{d t}\|u\|_{l+1}^{l+1}+\frac{l p^{p}}{(p+l-1)^{p}}\left\|\nabla u^{\frac{p+l-1}{p}}\right\|_{p}^{p}+\beta\|u\|_{l+1}^{l+1}=\lambda \int_{\Omega} u^{l}|\nabla u|^{r} d x
$$

Substitute (3.8) and (3.10) into the above equality we obtain

$$
\frac{1}{l+1} \frac{d}{d t}\|u\|_{l+1}^{l+1}+\left[\frac{l p^{p}}{(p+l-1)^{p}}-\lambda \varepsilon\right] C_{00}^{-p}\|u\|_{l+1}^{p+l-1}+\beta\|u\|_{l+1}^{l+1} \leq \lambda C(\varepsilon)|\Omega|^{\frac{p-2 r}{(l+1)(p-r)}}\|u\|_{l+1}^{l+\frac{r}{p-r}},
$$

i.e.

$$
\frac{d}{d t}\|u\|_{l+1}+\left[\frac{l p^{p}}{(p+l-1)^{p}}-\lambda \varepsilon\right] C_{00}^{-p}\|u\|_{l+1}^{p-1}+\beta\|u\|_{l+1} \leq \lambda C(\varepsilon)|\Omega|^{\frac{p-2 r}{(l+1)(p-r)}}\|u\|_{l+1}^{\frac{r}{p-r}} .
$$

By Lemma 3, there exists $\alpha_{2}>\beta$ such that

$$
0 \leq\|u\|_{l+1} \leq\left\|u_{0}\right\|_{l+1} e^{-\alpha_{2} t}, \quad t \geq 0,
$$

provided that

$$
\left\|u_{0}\right\|_{l+1}<\left[\frac{C_{00}^{-p}\left(\frac{l p^{p}}{(p+l-1)^{p}}-\lambda \varepsilon\right)}{\lambda C(\varepsilon)|\Omega|^{\frac{p-2 r}{(l+1)(p-r)}}}\right]^{\frac{1}{p-r}-p+1} .
$$

Furthermore, there exists $T_{6}>0$ such that

$$
\begin{aligned}
& {\left[\frac{l p^{p}}{(p+l-1)^{p}}-\lambda \varepsilon\right] C_{00}^{-p}-\lambda C(\varepsilon)|\Omega|^{\frac{p-2 r}{(l+1)(p-r)}}\|u\|_{l+1}^{\frac{r}{p-r}-p+1}} \\
& \quad \geq\left[\frac{l p^{p}}{(p+l-1)^{p}}-\lambda \varepsilon\right] C_{00}^{-p}-\lambda C(\varepsilon)|\Omega|^{\frac{p-2 r}{(l+1)(p-r)}}\left(\left\|u_{0}\right\|_{l+1} e^{-\alpha_{2} T_{6}}\right)^{\frac{r}{p-r}-p+1} \\
& \quad=C_{5}>0
\end{aligned}
$$

holds for $t \in\left[T_{6},+\infty\right)$. Therefore, when $t \in\left[T_{6},+\infty\right)$, we have

$$
\frac{d}{d t}\|u\|_{l+1}+C_{5}\|u\|_{l+1}^{p-1}+\beta\|u\|_{l+1} \leq 0 .
$$

By Lemma 2, we can obtain the desired decay estimate for

$$
T_{7}=\frac{1}{(2-p) \beta} \ln \left(1+\frac{\beta}{C_{5}}\left\|u\left(\cdot, T_{6}\right)\right\|_{l+1}^{2-p}\right)+T_{6} .
$$


(2) If $N=1$, multiplying (1.1) by $u^{k}$ and integrating over $\Omega$ yield

$$
\frac{1}{k+1} \frac{d}{d t}\|u\|_{k+1}^{k+1}+\frac{k p^{p}}{(k+p-1)^{p}}\left\|\nabla u^{\frac{p+k-1}{p}}\right\|_{p}^{p}+\beta\|u\|_{k+1}^{k+1}=\lambda \int_{\Omega} u^{k}|\nabla u|^{r} d x .
$$

If we substitute (3.8) and (3.14) into the above equality, we have as a result

$$
\begin{aligned}
& \frac{1}{k+1} \frac{d}{d t}\|u\|_{k+1}^{k+1}+\left[\frac{k p^{p}}{(k+p-1)^{p}}-\lambda \varepsilon\right] \gamma^{-p}\|u\|_{k+1}^{p+k-1}+\beta\|u\|_{k+1}^{k+1} \\
& \quad \leq \lambda C(\varepsilon)|\Omega|^{\frac{p-2 r}{(k+1)(p-r)}\|u\|_{k+1}^{k+\frac{r}{p-r}}}
\end{aligned}
$$

i.e.

$$
\frac{d}{d t}\|u\|_{k+1}+\left[\frac{k p^{p}}{(k+p-1)^{p}}-\lambda \varepsilon\right] \gamma^{-p}\|u\|_{k+1}^{p-1}+\beta\|u\|_{k+1} \leq \lambda C(\varepsilon)|\Omega|^{\frac{p-2 r}{(k+1)(p-r)}}\|u\|_{k+1}^{\frac{r}{p-r}}
$$

Here setting $k=1$ leads to

$$
\frac{d}{d t}\|u\|_{2}+(1-\lambda \varepsilon) \gamma^{-p}\|u\|_{2}^{p-1}+\beta\|u\|_{2} \leq \lambda C(\varepsilon)|\Omega|^{\frac{p-2 r}{2(p-r)}}\|u\|_{2}^{\frac{r}{p-r}}
$$

By Lemma 3, there exists $\alpha_{3}>\beta$ such that

$$
0 \leq\|u\|_{2} \leq\left\|u_{0}\right\|_{2} e^{-\alpha_{3} t}, \quad t \geq 0
$$

provided that

$$
\left\|u_{0}\right\|_{2}<\left[\frac{(1-\lambda \varepsilon) \gamma^{-p}}{\lambda C(\varepsilon)|\Omega|^{\frac{p-2 r}{2(p-r)}}}\right]^{\frac{1}{p-r}-p+1} .
$$

Furthermore, there exists $T_{8}>0$ such that

$$
\begin{aligned}
& (1-\lambda \varepsilon) \gamma^{-p}-\lambda C(\varepsilon)|\Omega|^{\frac{p-2 r}{2(p-r)}}\|u\|_{2}^{\frac{r}{p-r}-p+1} \\
& \quad \geq(1-\lambda \varepsilon) \gamma^{-p}-\lambda C(\varepsilon)|\Omega|^{\frac{p-2 r}{2(p-r)}}\left(\left\|u_{0}\right\|_{2} e^{-\alpha_{3} T_{8}}\right)^{\frac{r}{p-r}-p+1}=C_{6}>0
\end{aligned}
$$

holds for $t \in\left[T_{8},+\infty\right)$. Therefore, when $t \in\left[T_{8},+\infty\right)$, we have

$$
\frac{d}{d t}\|u\|_{2}+C_{6}\|u\|_{2}^{p-1}+\beta\|u\|_{2} \leq 0
$$

By Lemma 2, we can obtain the desired decay estimate for

$$
T_{9}=\frac{1}{(2-p) \beta} \ln \left(1+\frac{\beta}{C_{6}}\left\|u\left(\cdot, T_{8}\right)\right\|_{2}^{2-p}\right)+T_{8}
$$

\subsection{Proof of Theorem 3}

Let $v(x, t)=g(t) \phi(x)$, where $\phi(x)$ is the first eigenfunction corresponding to the first eigenvalue $\lambda_{1}$ for the homogeneous Dirichlet boundary value problem,

$$
-\operatorname{div}\left(|\nabla \phi|^{p-2} \nabla \phi\right)=\lambda|\phi|^{p-2} \phi, \quad x \in \Omega ; \quad \phi(x)=0, \quad x \in \partial \Omega,
$$


and let $\phi(x)$ satisfy $\phi(x)>0, \max _{x \in \Omega} \phi(x)=1$; by Lemma 4 , there exists one non-constant solution $g(t)$ that satisfies the ODE problem

$$
g^{\prime}(t)+\lambda_{1} g^{p-1}(t)+\beta g(t)=g^{r}(t), \quad t \geq 0 ; \quad g(0)=0, \quad g(t)>0, t>0 .
$$

Then for any test function $0 \leq \varphi(x, t) \in C_{0}^{\infty}\left(Q_{T}\right)$, we have

$$
\begin{aligned}
\int_{0}^{t} & \int_{\Omega}\left\{v_{s}(x, s) \varphi(x, s)-|\nabla v|^{p-2} \nabla \nu \nabla \varphi+\beta \nu(x, s) \varphi(x, s)-\lambda|\nabla \nu|^{r} \varphi(x, s)\right\} d x d s \\
= & \int_{0}^{t} \int_{\Omega}\left\{g^{\prime}(s) \phi(x)+\lambda_{1} g^{p-1}(s) \phi^{p-1}(x)+\beta g(s) \phi(x)-\lambda g^{r}(s)|\nabla \phi|^{r}\right\} \varphi(x, s) d x d s \\
= & \int_{0}^{t} \int_{\Omega}\left\{\left(-\lambda_{1} g^{p-1}(s)-\beta g(s)+g^{r}(s)\right) \phi(x)+\lambda_{1} g^{p-1}(s) \phi^{p-1}(x)\right. \\
& \left.+\beta g(s) \phi(x)-\lambda g^{r}(s)|\nabla \phi|^{r}\right\} \varphi(x, s) d x d s \\
\leq & \int_{0}^{t} \int_{\Omega}\left\{g^{r}(s) \phi(x)+\lambda_{1} g^{p-1}(s) \phi^{p-1}(x)-\lambda g^{r}(s)|\nabla \phi|^{r}\right\} \varphi(x, s) d x d s \\
\leq & \int_{0}^{t} \int_{\Omega}\left\{g^{r}(s)+\lambda_{1} g^{p-1}(s)-\lambda g^{r}(s)|\nabla \phi|^{r}\right\} \varphi(x, s) d x d s \\
= & \int_{0}^{t} \int_{\Omega} g^{r}(s)\left\{1+\lambda_{1} g^{p-1-r}(s)-\lambda|\nabla \phi|^{r}\right\} \varphi(x, s) d x d s .
\end{aligned}
$$

For such a $v(x, t)$ to be a subsolution of problem (1.1)-(1.3), it suffices to show that

$$
\int_{0}^{t} \int_{\Omega} g^{r}(s)\left\{1+\lambda_{1} g^{p-1-r}(s)-\lambda|\nabla \phi|^{r}\right\} \varphi(x, s) d x d s \leq 0 .
$$

Here we only show that

$$
\int_{\Omega}\left\{1+\lambda_{1} g^{p-1-r}(s)-\lambda|\nabla \phi|^{r}\right\} d x \leq 0 .
$$

Since for any $s \in(0, t), g(s)$ is bounded and for $p-1>r$, we find that there exists a positive constant $M>0$ such that

$$
0<g(s)<M \text {, }
$$

and

$$
0<g^{p-1-r}(s)<M^{p-1-r} .
$$

By choosing $\lambda \geq \frac{\left(1+\lambda_{1} M^{p-1-r}\right)|\Omega|}{\|\nabla \phi\|_{r}^{r}}$, we get

$$
\int_{\Omega}\left\{1+\lambda_{1} M^{p-1-r}-\lambda|\nabla \phi|^{r}\right\} d x \leq 0,
$$

which together with (3.24) implies that (3.23) holds. Moreover, $v(x, 0)=g(0) \phi(x)=0 \leq$ $u_{0}(x), x \in \Omega ; v(x, t)=0, x \in \partial \Omega, t>0$. Therefore, $v(x, t)$ is a non-extinction subsolution of 
problem (1.1)-(1.3). By the comparison principle, we have

$$
u(x, t) \geq v(x, t)>0, \quad x \in \Omega, t>0,
$$

which implies that the weak solution $u(x, t)$ of problem (1.1)-(1.3) cannot vanish in finite time.

4 The case $1<p<2,0<q<1$

\subsection{Proof of Theorem 4}

(1) If $N \geq 2$, we have the following.

(a) If $\frac{2 N}{N+2}<p<2$, multiplying (1.1) by $u$ and integrating over $\Omega$ yield

$$
\frac{1}{2} \frac{d}{d t}\|u\|_{2}^{2}+\|\nabla u\|_{p}^{p}+\beta\|u\|_{1+q}^{1+q}=\lambda \int_{\Omega} u|\nabla u|^{r} d x
$$

Since $p-1=r$, we easily get $\frac{r}{p-r}=p-1<1$. By the Young inequality, we obtain

$$
\begin{aligned}
\int_{\Omega} u|\nabla u|^{r} d x & \leq \varepsilon\|\nabla u\|_{p}^{p}+C(\varepsilon)|\Omega|^{\frac{p-2 r}{2(p-r)}}\|u\|_{1+\frac{r}{p-r}}^{1+\frac{r}{p-r}} \\
& =\varepsilon\|\nabla u\|_{p}^{p}+C(\varepsilon)|\Omega|^{\frac{1-r}{2}}\|u\|_{p}^{p} .
\end{aligned}
$$

We substitute (4.2) into (4.1) to obtain

$$
\frac{1}{2} \frac{d}{d t}\|u\|_{2}^{2}+\left[1-\lambda \varepsilon-\frac{\lambda C(\varepsilon)|\Omega|^{\frac{1-r}{2}}}{\lambda_{1}}\right]\|\nabla u\|_{p}^{p}+\beta\|u\|_{1+q}^{1+q} \leq 0 .
$$

By the Gagliardo-Nirenberg inequality, we have

$$
\|u\|_{2} \leq C(N, p, q)\|u\|_{1+q}^{1-\theta_{1}}\|\nabla u\|_{p}^{\theta_{1}},
$$

where $\theta_{1}=\left(\frac{1}{1+q}-\frac{1}{2}\right)\left(\frac{1}{N}-\frac{1}{p}+\frac{1}{1+q}\right)^{-1}=\frac{N p(1-q)}{2 p(q+1)+2 N(p-q-1)}$. Since $\frac{2 N}{N+2}<p<2$ and $0<q<1$, we easily get $0<\theta_{1}<1$. By the Young inequality again, we have

$$
\begin{aligned}
\|u\|_{2}^{k_{1}} & \leq C(N, p, q)^{k_{1}}\|u\|_{1+q}^{k_{1}\left(1-\theta_{1}\right)}\|\nabla u\|_{p}^{k_{1} \theta_{1}}, \\
& \leq C(N, p, q)^{k_{1}}\left(\eta_{1}\|\nabla u\|_{p}^{p}+C\left(\eta_{1}\right)\|u\|_{1+q}^{\frac{k_{1} p\left(1-\theta_{1}\right)}{p-k_{1} \theta_{1}}}\right),
\end{aligned}
$$

where $\eta_{1}>0, k_{1}>1$ will be determined later. Here we set $k_{1}=\frac{p(1+q)}{p\left(1-\theta_{1}\right)+\theta_{1}(1+q)}=$ $\frac{2 p(q+1)+2 N(p-q-1)}{2 p+N(p-q-1)}$, then we have $1<k_{1}<2$ and $\frac{k_{1} p\left(1-\theta_{1}\right)}{p-k_{1} \theta_{1}}=1+q$, and

$$
\frac{C(N, p, q)^{-k_{1}} \beta}{C\left(\eta_{1}\right)}\|u\|_{2}^{k_{1}} \leq \frac{\eta_{1} \beta}{C\left(\eta_{1}\right)}\|\nabla u\|_{p}^{p}+\beta\|u\|_{1+q}^{1+q}
$$

and we now substitute (4.4) into (4.3) to obtain

$$
\frac{1}{2} \frac{d}{d t}\|u\|_{2}^{2}+\left[1-\lambda \varepsilon-\frac{\lambda C(\varepsilon, r, p)|\Omega|^{\frac{1-r}{2}}}{\lambda_{1}}-\frac{\eta_{1} \beta}{C\left(\eta_{1}\right)}\right]\|\nabla u\|_{p}^{p}+\frac{C(N, p, q)^{-k_{1}} \beta}{C\left(\eta_{1}\right)}\|u\|_{2}^{k_{1}} \leq 0
$$


Here we can choose $\lambda$ small enough such that $1-\lambda \varepsilon-\frac{\lambda C(\varepsilon, r, p)|\Omega|^{\frac{1-r}{2}}}{\lambda_{1}}-\frac{\eta_{1} \beta}{C\left(\eta_{1}\right)} \geq 0$. Here setting $C_{7}=\frac{C(N, p, q)^{-k} k_{1} \beta}{C\left(\eta_{1}\right)}$ leads to

$$
\frac{d}{d t}\|u\|_{2}+C_{7}\|u\|_{2}^{k_{1}-1} \leq 0
$$

By Lemma 1, we have

$$
\begin{aligned}
\|u\|_{2} & \leq\left[\left\|u_{0}\right\|_{2}^{2-k_{1}}-C_{7}\left(2-k_{1}\right) t\right]^{\frac{1}{2-k_{1}}}, \quad t \in\left[0, T_{10}\right), \\
\|u\|_{2} & \equiv 0, \quad t \in\left[T_{10},+\infty\right)
\end{aligned}
$$

where $T_{10}=\frac{\left\|u_{0}\right\|_{2}^{2-k_{1}}}{C_{7}\left(2-k_{1}\right)}$.

(b) If $1<p \leq \frac{2 N}{N+2}$, multiplying (1.1) by $u^{s}$ (where $s>\frac{2 N-(N+1) p}{p} \geq 1$ ) and integrating over $\Omega$ yield

$$
\frac{1}{s+1} \frac{d}{d t}\|u\|_{s+1}^{s+1}+\frac{s p^{p}}{(p+s-1)^{p}}\left\|\nabla u^{\frac{p+s-1}{p}}\right\|_{p}^{p}+\beta\|u\|_{s+q}^{s+q}=\lambda \int_{\Omega} u^{s}|\nabla u|^{r} d x
$$

By the Young inequality, we have

$$
\begin{aligned}
\int_{\Omega} u^{s}|\nabla u|^{r} d x & =\left(\frac{p+s-1}{p}\right)^{r} \int_{\Omega} u^{s-\frac{(s-1) r}{p}}\left|\nabla u^{\frac{p+s-1}{p}}\right|^{r} d x \\
& \leq \varepsilon\left\|\nabla u^{\frac{p+s-1}{p}}\right\|_{p}^{p}+C(\varepsilon) \int_{\Omega} u^{s+\frac{r}{p-r}} d x \\
& =\varepsilon\left\|\nabla u^{\frac{p+s-1}{p}}\right\|_{p}^{p}+C(\varepsilon)\|u\|_{s+p-1}^{s+p-1} .
\end{aligned}
$$

By the Gagliardo-Nirenberg inequality, we have

$$
\|u\|_{s+1} \leq C(N, p, q, s)\|u\|_{s+q}^{1-\theta_{2}}\left\|\nabla u^{\frac{p+s-1}{p}}\right\| \frac{\theta_{2} p}{p+s-1},
$$

where $\theta_{2}=\frac{p+s-1}{p}\left(\frac{1}{s+q}-\frac{1}{s+1}\right)\left(\frac{1}{N}-\frac{1}{p}+\frac{p+s-1}{p} \cdot \frac{1}{s+q}\right)^{-1}=\frac{N(p+s-1)(1-q)}{(s+1)[p(q+s)+N(p-q-1)]}$. By the choice of $s$, we get $0<\theta_{2}<1$. By the Young inequality again, we obtain

$$
\begin{aligned}
\|u\|_{s+1}^{k_{2}} & \leq C(N, p, q, s)^{k_{2}}\|u\|_{s+q}^{k_{2}\left(1-\theta_{2}\right)}\left\|\nabla u^{\frac{p+s-1}{p}}\right\|_{p}^{\frac{k_{2} \theta_{2} p}{p+s-1}} \\
& \leq C(N, p, q, s)^{k_{2}}\left(\eta_{2}\left\|\nabla u^{\frac{p+s-1}{p}}\right\|_{p}^{p}+C\left(\eta_{2}\right)\|u\|_{s+q}^{\frac{k_{2}\left(1-\theta_{2}\right)(p+s-1)}{p+s-1-k_{2} \theta_{2}}}\right),
\end{aligned}
$$

where $\eta_{2}>0, k_{2}>0$ will be determined later. Here we set $k_{2}=\frac{(p+s-1)(s+q)}{(p+s-1)\left(1-\theta_{2}\right)+\theta_{2}(s+q)}=$ $\frac{(s+1)[p(q+s)+N(p-q-1)]}{p(s+1)+N(p-q-1)}$, then we have $s<k_{2}<s+1$ and $\frac{k_{2}\left(1-\theta_{2}\right)(p+s-1)}{p+s-1-k_{2} \theta_{2}}=s+q$, and

$$
\frac{C(N, p, q, s)^{-k_{2}} \beta}{C\left(\eta_{2}\right)}\|u\|_{s+1}^{k_{2}} \leq \frac{\eta_{2} \beta}{C\left(\eta_{2}\right)}\left\|\nabla u^{\frac{p+s-1}{p}}\right\|_{p}^{p}+\beta\|u\|_{s+q}^{s+q},
$$


and we now substitute (4.6) into (4.5) to obtain

$$
\begin{aligned}
& \frac{1}{s+1} \frac{d}{d t}\|u\|_{s+1}^{s+1}+\left[\frac{s p^{p}}{(p+s-1)^{p}}-\lambda \varepsilon-\frac{\lambda C(\varepsilon)}{\lambda_{1}}-\frac{\eta_{2} \beta}{C\left(\eta_{2}\right)}\right]\left\|\nabla u^{\frac{p+s-1}{p}}\right\|_{p}^{p} \\
& +\frac{C(N, p, q, s)^{-k_{2}} \beta}{C\left(\eta_{2}\right)}\|u\|_{s+1}^{k_{2}} \leq 0 .
\end{aligned}
$$

Here we can choose $\lambda$ small enough such that $\frac{s p^{p}}{(p+s-1)^{p}}-\lambda \varepsilon-\frac{\lambda C(\varepsilon)}{\lambda_{1}}-\frac{\eta_{2} \beta}{C\left(\eta_{2}\right)} \geq 0$. Setting $C_{8}=\frac{C(N, p, q,)^{-k_{2}} \beta}{C\left(\eta_{2}\right)}>0$, thus we have

$$
\frac{d}{d t}\|u\|_{s+1}+C_{8}\|u\|_{s+1}^{k_{2}-s} \leq 0
$$

By Lemma 1, we can obtain the desired decay estimate.

(2) If $N=1$, the proof is similar to the proof of (1)(a) except for using the GagliardoNirenberg inequality in the lower dimensional space, and we omit it here.

\subsection{Proof of Theorem 5}

(1) If $N \geq 2$, we have the following.

(a) If $\frac{2 N}{N+2}<p<2$, multiplying (1.1) by $u$ and integrating over $\Omega$ yield

$$
\frac{1}{2} \frac{d}{d t}\|u\|_{2}^{2}+\|\nabla u\|_{p}^{p}+\beta\|u\|_{1+q}^{1+q}=\lambda \int_{\Omega} u|\nabla u|^{r} d x
$$

Substituting (4.4) into the above equality gives

$$
\frac{1}{2} \frac{d}{d t}\|u\|_{2}^{2}+\left[1-\lambda \varepsilon-\frac{\eta_{1} \beta}{C\left(\eta_{1}\right)}\right]\|\nabla u\|_{p}^{p}+\frac{C(N, p, q)^{-k_{1}} \beta}{C\left(\eta_{1}\right)}\|u\|_{2}^{k_{1}} \leq \lambda C(\varepsilon)|\Omega|^{\frac{p-2 r}{2(p-r)}}\|u\|_{2}^{1+\frac{r}{p-r}} .
$$

Here we can choose $\varepsilon$ (or $\eta_{1}$ ) small enough such that $1-\lambda \varepsilon-\frac{\eta_{1} \beta}{C\left(\eta_{1}\right)} \geq 0$, thus we get

$$
\frac{d}{d t}\|u\|_{2}+\|u\|_{2}^{k_{1}-1}\left[\frac{C(N, p, q)^{-k_{1}} \beta}{C\left(\eta_{1}\right)}-\lambda C(\varepsilon)|\Omega|^{\frac{p-2 r}{2(p-r)}}\|u\|_{2}^{\frac{r}{p-r}-k_{1}+1}\right] \leq 0 .
$$

Therefore,

$$
\frac{d}{d t}\|u\|_{2}+C_{9}\|u\|_{2}^{k_{1}-1} \leq 0
$$

provided that

$$
\left\|u_{0}\right\|_{2}<\left[\frac{C(N, p, q)^{-k_{1}} \beta}{C\left(\eta_{1}\right) \lambda C(\varepsilon)|\Omega|^{\frac{p-2 r}{2(p-r)}}}\right]^{\frac{1}{p-r-k}+1},
$$

and

$$
\frac{r}{p-r}>k_{1}-1
$$


i.e.

$$
r>\frac{2 p^{2} q+N p(p-q+1)}{2 p(q+1)+2 N(p-q+1)}
$$

where

$$
C_{9}=\frac{C(N, p, q)^{-k_{1}} \beta}{C\left(\eta_{1}\right)}-\lambda C(\varepsilon)|\Omega|^{\frac{p-2 r}{2(p-r)}}\left\|u_{0}\right\|_{2}^{\frac{r}{p-r}-k_{1}+1}>0 .
$$

By Lemma 1, we can obtain the desired decay estimate. Since $p>\frac{2 N}{N+2}$, we have $2 p>N(2-$ $p$ ). Therefore, if $q \geq p-1$, then $r>p-1$.

(b) If $1<p \leq \frac{2 N}{N+2}$, multiplying (1.1) by $u^{s}$ (where $s>\frac{2 N-(N+1) p}{p} \geq 1$ ) and integrating over $\Omega$ yield

$$
\frac{1}{s+1} \frac{d}{d t}\|u\|_{s+1}^{s+1}+\frac{s p^{p}}{(p+s-1)^{p}}\left\|\nabla u^{\frac{p+s-1}{p}}\right\|_{p}^{p}+\beta\|u\|_{s+q}^{s+q}=\lambda \int_{\Omega} u^{s}|\nabla u|^{r} d x
$$

Substituting (4.6) into the above equality gives

$$
\begin{aligned}
& \frac{1}{s+1} \frac{d}{d t}\|u\|_{s+1}^{s+1}+\left[\frac{s p^{p}}{(p+s-1)^{p}}-\lambda \varepsilon-\frac{\eta_{2} \beta}{C\left(\eta_{2}\right)}\right]\left\|\nabla u^{\frac{p+s-1}{p}}\right\|_{p}^{p}+\frac{C(N, p, q, s)^{-k_{2}} \beta}{C\left(\eta_{2}\right)}\|u\|_{s+1}^{k_{2}} \\
& \quad \leq \lambda C(\varepsilon)|\Omega|^{\frac{p-2 r}{(s+1)(p-r)}\|u\|_{s+1}^{s+\frac{r}{p-r}}} .
\end{aligned}
$$

Here we can choose $\varepsilon$ (or $\left.\eta_{1}\right)$ small enough such that $\frac{s p^{p}}{(p+s-1)^{p}}-\lambda \varepsilon-\frac{\eta_{2} \beta}{C\left(\eta_{2}\right)} \geq 0$, thus we get

$$
\frac{d}{d t}\|u\|_{s+1}+\|u\|_{s+1}^{k_{2}-s}\left[\frac{C(N, p, q, s)^{-k_{2}} \beta}{C\left(\eta_{2}\right)}-\lambda C(\varepsilon)|\Omega|^{\frac{p-2 r}{(s+1)(p-r)}}\left\|u_{0}\right\|_{s+1}^{\frac{r}{p-r}-k_{2}+s}\right] \leq 0 .
$$

Therefore,

$$
\frac{d}{d t}\|u\|_{s+1}+C_{10}\|u\|_{s+1}^{k_{2}-s} \leq 0,
$$

provided that

$$
\left\|u_{0}\right\|_{s+1}<\left[\frac{C(N, p, q, s)^{-k_{2}} \beta}{C\left(\eta_{2}\right) \lambda C(\varepsilon)|\Omega|^{\frac{p-2 r}{(s+1)(p-r)}}}\right]^{\frac{1}{p-r}-k_{2}+s},
$$

and

$$
\frac{r}{p-r}>k_{2}-s,
$$

i.e.

$$
r>\frac{p^{2} q(s+1)+N p(p-q-1)}{p(s+1)(1+q)+2 N(p-q-1)},
$$

where

$$
C_{10}=\frac{C(N, p, q, s)^{-k_{2}} \beta}{C\left(\eta_{2}\right)-\lambda C(\varepsilon)|\Omega|^{\frac{p-2 r}{(s+1)(p-r)}}}\left\|u_{0}\right\|_{s+1}^{\frac{r}{p-r}-k_{2}+s}>0 .
$$


By Lemma 1, we can obtain the desired decay estimate. Since $s>\frac{2 N-(N+1) p}{p}$, it follows that $p(s+1)>N(2-p)$. Therefore, if $q \geq p-1$, then $r>p-1$.

(2) If $N=1$, the proof is similar to the proof of (1)(a) except for using the GagliardoNirenberg inequality in the lower dimensional space, and we omit it here.

Competing interests

The authors declare that they have no competing interests.

\section{Authors' contributions}

All authors contributed equally to the manuscript and read and approved the final manuscript.

\section{Author details}

'Department of Mathematics, Pusan National University, Busan, 609-735, Republic of Korea. ${ }^{2}$ School of Mathematical Sciences, Ocean University of China, Qingdao, 266100, P.R. China.

\section{Acknowledgements}

This work is supported by the Natural Science Foundation of Shandong Province of China (ZR2012AM018) and the Fundamental Research Funds for the Central Universities (No. 201362032). The authors would like to warmly thank all the reviewers for their insightful and constructive comments.

Received: 7 October 2013 Accepted: 24 January 2014 Published: 12 Feb 2014

\section{References}

1. Kawohl, B: On a family of torsional creep problems. J. Reine Angew. Math. 410, 1-22 (1990)

2. Showalter, RE, Walkington, NJ: Diffusion of fluid in a fissured medium with microstructure. SIAM J. Math. Anal. 22, 1702-1722 (1991)

3. Pélissier, MC, Reynaud, ML: Étude d'un modèle mathématique d'écoulement de glacier. C. R. Acad. Sci., Ser. 1 Math. 279, 531-534 (1974)

4. Kalashnikov, AS: The propagation of disturbances in problem of nonlinear heat conduction with absorption. USSR Comput. Math. Math. Phys. 14, 70-85 (1974)

5. Dibenedetto, E: Degenerate Parabolic Equations. Springer, New York (1993)

6. Yuan, HJ, Xu, XJ, Gao, WJ, Lian, SZ, Cao, CL: Extinction and positivity for the evolution $p$-Laplacian equation with $L$ initial value. J. Math. Anal. Appl. 310, 328-337 (2005)

7. Gu, YG: Necessary and sufficient conditions of extinction of solution on parabolic equations. Acta Math. Sin. 37, 73-79 (1994) (in Chinese)

8. Tian, Y, Mu, CL: Extinction and non-extinction for a $p$-Laplacian equation with nonlinear source. Nonlinear Anal. TMA 69, 2422-2431 (2008)

9. Yin, JX, Jin, CH: Critical extinction and blow-up exponents for fast diffusive $p$-Laplacian with sources. Math. Methods Appl. Sci. 30, 1147-1167 (2007)

10. Fang, ZB, Li, G: Extinction and decay estimates of solutions for a class of doubly degenerate equations. Appl. Math. Lett. 25, 1795-1802 (2012)

11. Fang, ZB, Xu, XH: Extinction behavior of solutions for the $p$-Laplacian equations with nonlocal sources. Nonlinear Anal., Real World Appl. 13, 1780-1789 (2012)

12. $\mathrm{Xu}, \mathrm{XH}$, Fang, ZB, Yi, SC: Extinction and decay estimates of solutions for porous medium equation with nonlocal source and strong absorption. Bound. Value Probl. 2013, Article ID 13 (2013). doi:10.1186/1687-2770-2013-24

13. Benachour, S, Dabuleanu, S, Laurencot, P: Decay estimates for a viscous Hamilton-Jacobi equation with homogeneous Dirichlet boundary conditions. Asymptot. Anal. 51, 209-229 (2007)

14. Lagar, RG, Laurencot, P: Positivity, decay, and extinction for a singular diffusion equation with gradient absorption. J. Funct. Anal. 262, 3186-3239 (2012)

15. $\mathrm{Mu}, \mathrm{CL}$, Yan, L, Xiao, YB: Extinction and non-extinction for the fast diffusion equation. Abstr. Appl. Anal. 2013, Article ID $747613(2013)$

16. Yang, SX: Extinction of solutions for evolution $p$-Laplacian equations with a gradient term. J. Xiamen Univ. (Nat. Sci.) 38, 811-815 (1999) (in Chinese)

17. Ohnuma, M, Sato, K: Singular degenerate parabolic equations with applications to the $p$-Laplace diffusion equation Commun. Partial Differ. Equ. 22, 381-411 (1997)

18. Ladyzenska, OA, Solonnikav, VA, Vral'tseva, NN: Linear and Quasilinear Equations of Parabolic Type. Am. Math. Soc, Providence (1968) 